\title{
Catheter Ablation of Atrial Fibrillation
}

\author{
- State of the Art -
}

\author{
Koichiro Kumagai, MD
}

\begin{abstract}
Atrial fibrillation (AF) is an arrhythmia associated with increased morbidity and mortality. Since the first report of catheter ablation curing AF, numerous techniques have evolved, from linear ablation to segmental pulmonary vein (PV) isolation, to extensive encircling PV isolation, to left atrial (LA) linear ablation, to ablation of complex fragmented atrial electrograms (CFAEs) and ablation of ganglionated plexi. A new approach for complete isolation of the posterior LA, including all PVs, is box isolation. $\mathrm{PV}$ isolation is associated with a high clinical success rate in paroxysmal AF. However, in persistent AF or longstanding persistent AF, PV isolation only may not be sufficient, so additional ablation at sites with CFAEs is needed to improve the clinical outcome. A hybrid approach of combining PV isolation plus CFAE ablation is highly effective in the majority of patients with persistent AF or longstanding persistent AF. Thus, AF ablation is an effective and established treatment for AF that offers an excellent chance of a lasting cure. It is about time that AF ablation became a first-line therapy for selected patients with AF. (Circ J 2011; 75: 2305-2311)
\end{abstract}

Key Words: Atrial fibrillation; Catheter ablation; Pulmonary veins

A trial fibrillation (AF) is the most common sustained cardiac arrhythmia that affects patient morbidity and mortality. Catheter ablation for AF has emerged as a promising new treatment strategy. In contrast to antiarrhythmic drugs, catheter ablation directly eliminates the inciting factors for AF and offers the possibility of a lasting cure. Various methods of ablating AF have been tried. Creating linear lesions in the atria mimicked the surgical Maze procedure. However, after recognizing the limited success and risks associated with this approach, Haissaguerre et $\mathrm{al}^{1}$ and Chen et $\mathrm{al}^{2}$ found that most focal AF is initiated by premature beats from the orifices of the pulmonary veins (PVs) or from the myocardial sleeves inside the PVs, and radiofrequency (RF) catheter ablation of triggered foci has been shown to cure AF. This discovery created a new era in the treatment of AF. The initial approach was focal ablation of the culprit PV identified as the triggering site initiating AF. However, detecting the exact focus is often difficult when atrial premature beats are infrequent, or each extrasystole may induce AF, thus necessitating repeated defibrillation. For these reasons, an alternative approach that simply seeks to electrically isolate the PVs from the left atrium (LA) at electrophysiological breakthroughs from the LA to the PV was provided. ${ }^{3}$ Moreover, numerous techniques have evolved, from segmental PV isolation to extensive encircling PV isolation, ${ }^{4}$ to LA linear ablation, ${ }^{5}$ box isolation, ${ }^{6}$ ablation of complex fragmented atrial electrograms (CFAEs) ${ }^{7}$ and ablation of ganglionated plexi. ${ }^{8}$

\section{Electrophysiology of the PV}

As early as the 1960s, detailed conduction and arrhythmic properties in the thoracic veins and their junctions with the atria were described by Japanese investigators. ${ }^{9}$ Despite these very early observations, investigation of the anatomic and electrophysiologic properties of the PVs remained limited, until the importance of PV triggers in the development of AF was appreciated. PV focal firing by abnormal automaticity and triggered activity may trigger $\mathrm{AF}$ or act as a rapid driver to maintain AF. Other experimental studies have provided evidence that suggests the PVs and the posterior LA are also preferred sites for reentrant arrhythmias. ${ }^{10-12}$ My group also demonstrated that the PV-LA junction has heterogeneous electrophysiological properties capable of sustaining reentry in humans. ${ }^{13}$ In a clinical study using basket catheter mapping, a PV-LA unstable reentrant circuit involving the exit and entrance breakthrough points at the PV-LA junction was observed. The different conduction properties of the exit and entrance sites, depending on the site of discharge, may contribute to reentry formation. The presence of anisotropic structures at the PV-LA junction may be critical for reentry. Thus, the PVs play a critical role in both triggering and maintaining AF.

\section{PV Isolation}

The goals of AF ablation procedures are to prevent $\mathrm{AF}$ by either eliminating the trigger that initiates $\mathrm{AF}$ or by altering

Received June 26, 2011; revised manuscript received August 12, 2011; accepted August 16, 2011; released online September 13, 2011

Heart Rhythm Center, Fukuoka Sanno Hospital, Fukuoka, Japan

Mailing address: Koichiro Kumagai, MD, Heart Rhythm Center, Fukuoka Sanno Hospital, 3-6-45 Momochihama, Sawara-ku, Fukuoka

814-0001, Japan. E-mail: kumagai@ kouhoukai.or.jp

ISSN-1346-9843 doi:10.1253/circj.CJ-11-0658

All rights are reserved to the Japanese Circulation Society. For permissions, please e-mail: cj@j-circ.or.jp 

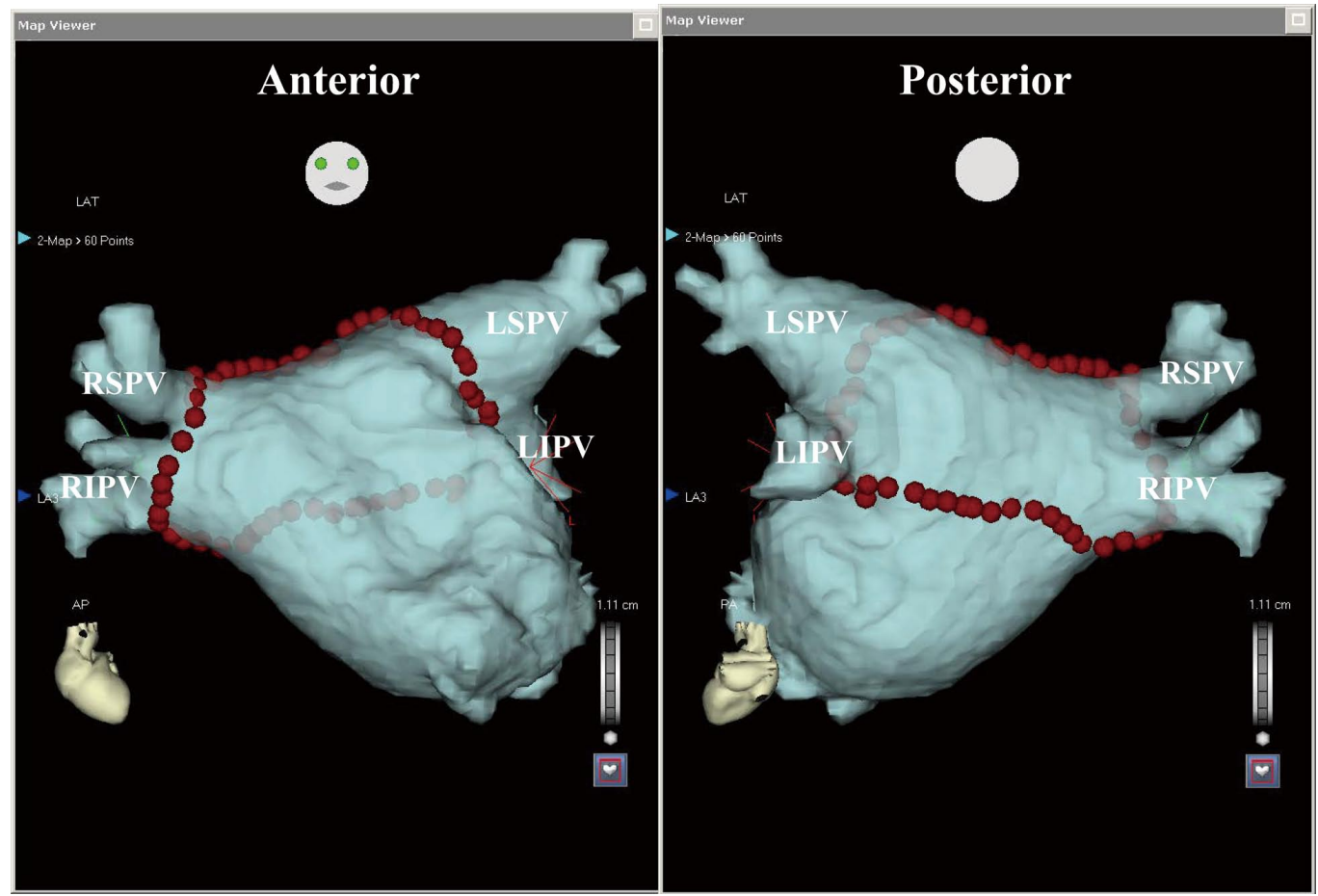

Figure 1. Box isolation. LSPV, left superior pulmonary vein; LIPV, left inferior pulmonary vein; RSPV, right superior pulmonary vein; RIPV, right inferior pulmonary vein.

the arrhythmogenic substrate. A recent consensus of worldrenowned experts in AF ablation states that PV isolation is a cornerstone of catheter ablation of AF, and most laboratories perform $\mathrm{PV}$ isolation as the primary approach for patients with AF. Earlier studies of electrophysiology-guided segmental ablation at the ostial level suggested that PV disconnection could be achieved with minimal ablation by targeting specific breakthrough points between the PV and LA., ${ }^{3,14}$ Complete electrical isolation was typically achieved after $\sim 50 \%$ of the circumference of the PV ostium was ablated. ${ }^{14}$ However, PV ostial ablation may result in PV stenosis. ${ }^{15,16}$ Therefore, most operators have moved toward ablation away from the PV ostium toward the level of the antrum. ${ }^{17-19}$ The antrum blends into the posterior wall of the LA. To encompass as much of the PV structure as possible, ablation needs to be performed around the entire antrum, along the posterior LA wall. ${ }^{18,19}$ The circumferential lesions may also alter the arrhythmogenic substrate by eliminating tissue located near the PV-LA junction that provides a substrate for reentrant circuits that may generate or perpetuate $\mathrm{AF}$, and/or by reducing the mass of atrial tissue needed to sustain reentry.

A recent meta-analysis of 31 studies including 2,800 patients found that the single-procedure success rate of PV isolation of all types of AF without antiarrhythmic drugs was $57 \%$ $(50-64 \%) \cdot{ }^{20}$ An analysis of 34 studies enrolling a total of 3,481 patients shows that the success rate without antiarrhythmic drugs increased to $71 \%(65-77 \%)$ after multiple procedures. ${ }^{20}$ However, examining data from 6 pioneering centers with greater experience in $\mathrm{AF}$ ablation, the success rate without antiarrhythmic drugs was $81 \%(71-88 \%)$ in 1,039 patients followed up for a period of 6 months to 2.4 years. $^{21}$

\section{Box Isolation}

Both the PVs and the posterior LA develop from the sinus venosus, where there are many pacemaker cells with spontaneous rhythmic activity in the early embryonic heart. ${ }^{22}$ The discrete site of high-frequency periodic activity is localized most often to the posterior LA, including the PV, during AF in sheep hearts. ${ }^{23}$ Non-PV foci originated mainly from the $\mathrm{PV}$ ostium or from the posterior LA, ${ }^{24}$ and the posterior LA and the LA roof serve as a substrate for maintenance of AF in patients with AF. ${ }^{5,25}$ It has been proposed that the surgical procedures for isolating the posterior LA and PVs could cure $\mathrm{AF}$ in $93 \%$ of patients with lone $\mathrm{AF}^{26}$ and $86 \%$ with chronic $A F{ }^{27}$ These findings support that isolation of not only the PVs but also the whole posterior LA can result in a much better cure rate in the patients with paroxysmal and persistent AF. Therefore, a new approach for complete isolation of the posterior LA, including all PVs, has been developed, namely, box isolation (Figure 1). ${ }^{6}$ In the posterior LA, there are many arrhythmogenic substrates for AF, including the triggers, reentries, and ganglionated plexi. Box isolation can contain these abnormal substrates in the posterior LA. There is increasing recognition of the importance of atrial autonomic ganglia in $\mathrm{AF}$ maintenance and the value of targeting them in AF ablation procedures. ${ }^{28}$ The lesion set of box isolation may interrupt sympathetic and parasympathetic denervation from the autonomic ganglia, which have been identified as potential triggers for AF. ${ }^{29,30}$

Techniques and Endpoints for Box Isolation Box isolation is performed by my group as the primary approach for patients with all types of AF. Radiofrequency energy is delivered with a power of $30 \mathrm{~W}$ using an irrigated-tip ablation catheter. The temperature is limited to $40^{\circ} \mathrm{C}$. The operator should pay atten- 

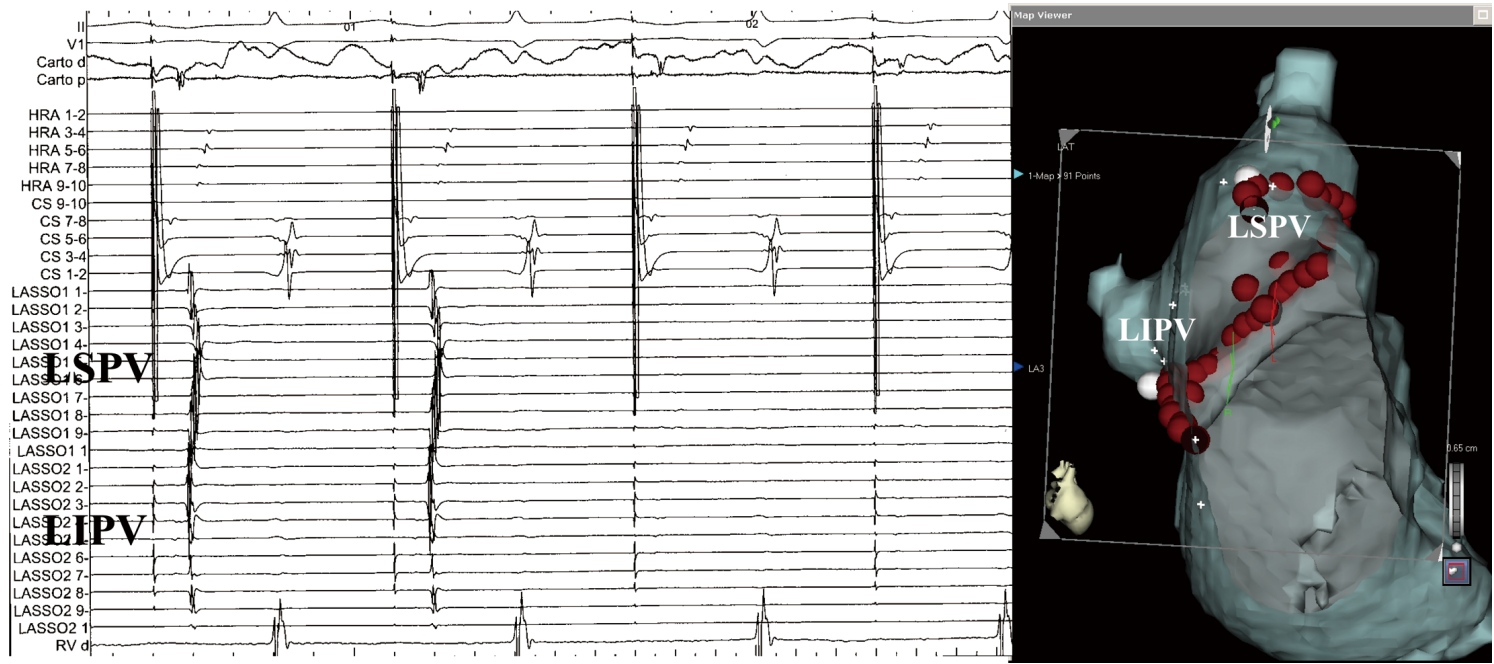

Figure 2. Isolation of pulmonary veins (PV). (Left) Tracings of electrograms during ablation. Lasso 1 is positioned in the left superior PV (LSPV) and Lasso 2 in the left inferior PV (LIPV). (Right) Inside view of the left PVs. In this case the left PVs were not isolated by only anterior lines. Segmental ablation of the breakthrough points at the carina created simultaneous isolation of the left ipsilateral PVs.

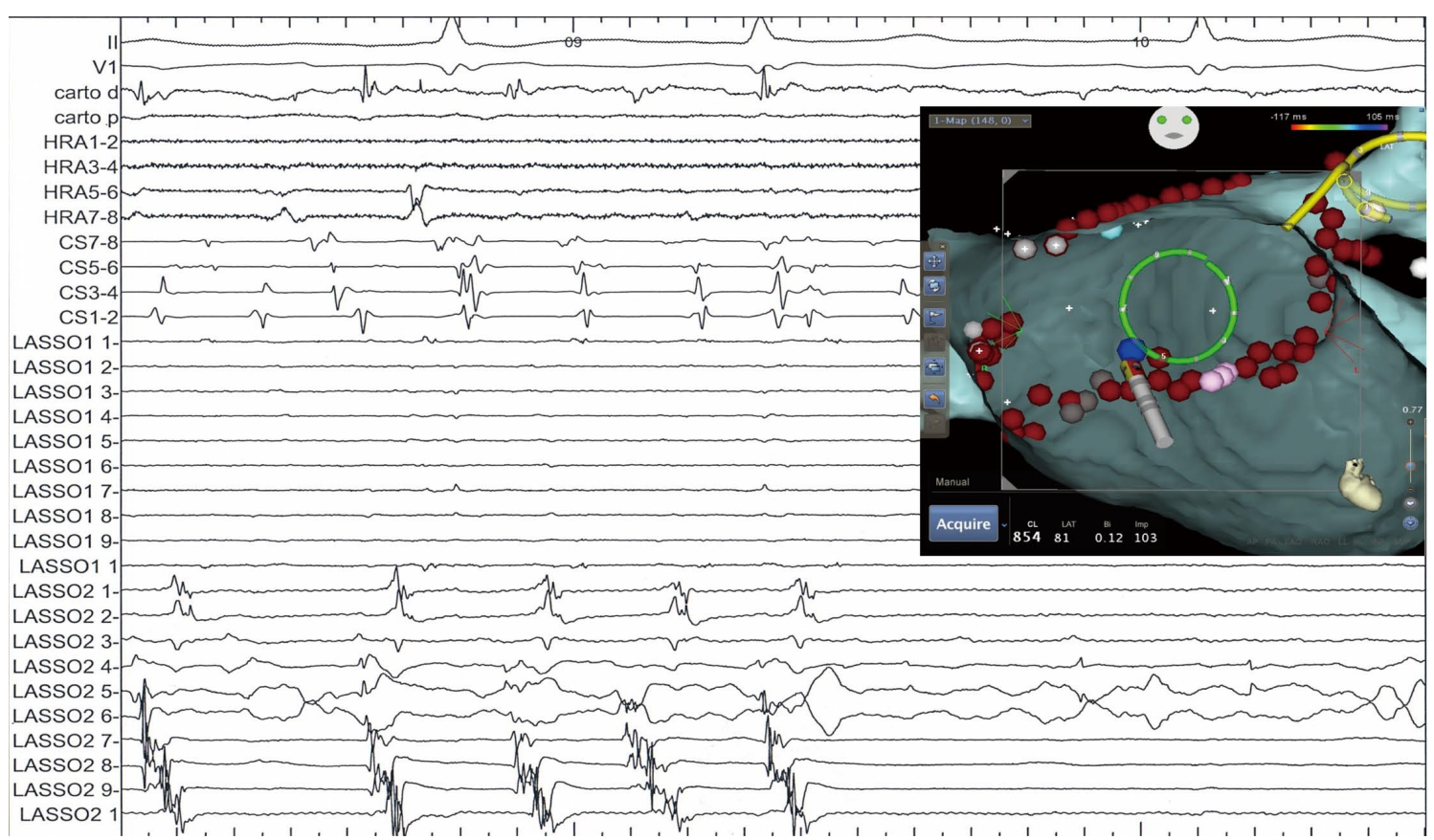

Figure 3. Entrance block of the box lesion. Lasso 1 is positioned in the LSPV (yellow) and Lasso 2 is positioned at the posterior wall within the box (green). Ablation of a gap at the mid floor line (Lasso 6) created the entrance block of the box lesion during atrial fibrillation. LSPV, left superior pulmonary vein.

tion to ablating the esophageal aspect of the floorline by decreasing the power and duration of RF energy application. Therefore, the luminal esophageal temperature is monitored with a catheter in the esophagus close to the tip of the ablation catheter. During the ablation at the posterior LA close to the esophagus, cooling water through a tube just above the catheter is infused into the esophagus and the ablation is performed at a maximum power of $20 \mathrm{~W}$ and a temperature of $40^{\circ} \mathrm{C}$. If the esophageal temperature is higher than $38^{\circ} \mathrm{C}, \mathrm{RF}$ applications are interrupted. Radiofrequency energy is delivered for $30 \mathrm{~s}$ at each point.

Continuous lesions at the anterior portion of the ipsilateral 


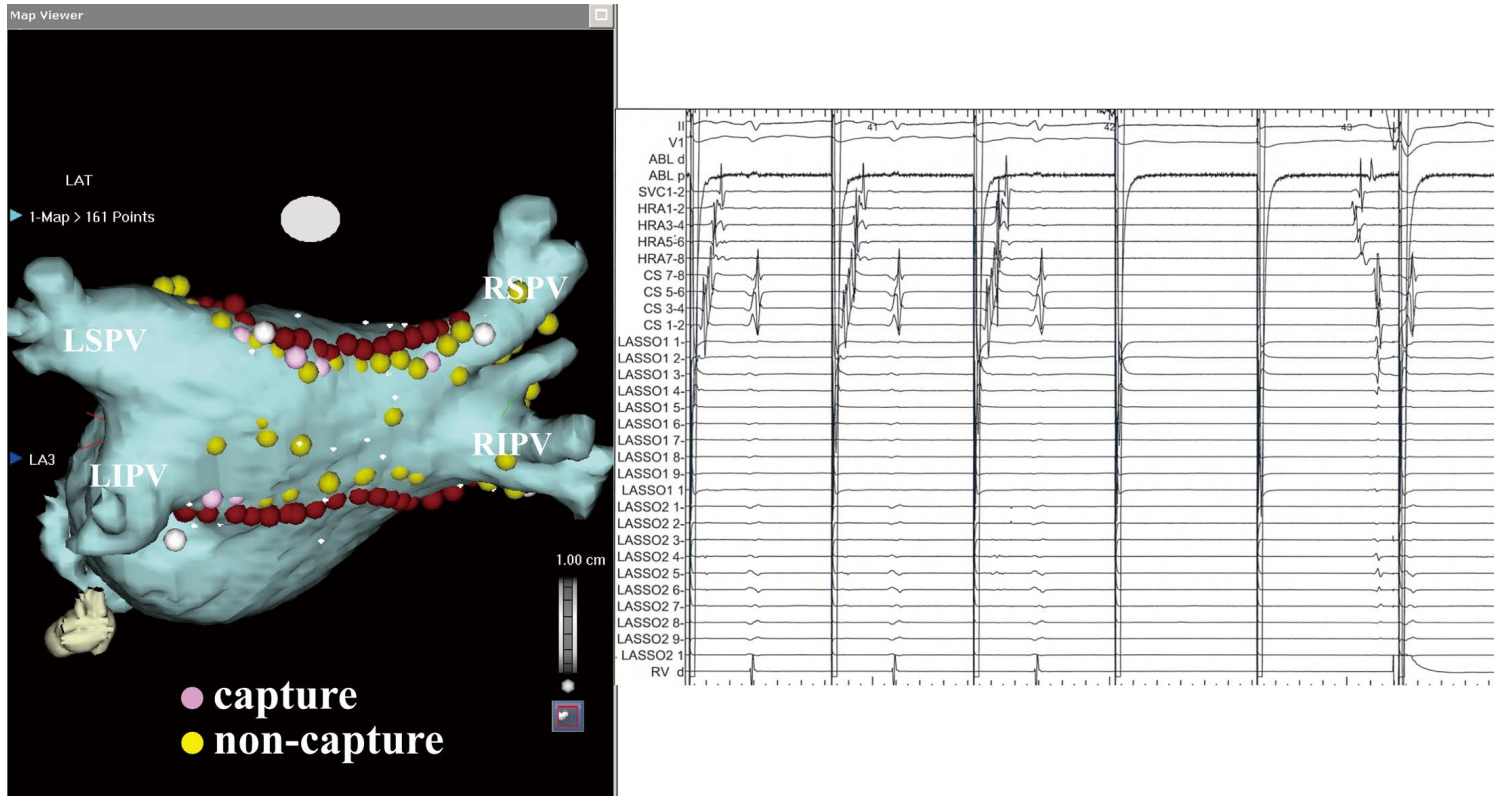

Figure 4. Exit block of the box lesion. (Left) During sinus rhythm, gaps along the ablation lines were detected and closed using high voltage $(10 \mathrm{~V})$ pace mapping through the ablation catheter. With lack of left atrial (LA) capture (yellow), the line was considered as complete at this location. In the case of LA capture (pink), a gap was suspected and RF energy was delivered simultaneously while pacing from the tip of the ablation catheter. (Right) Ablation at the site with LA capture was continued until the was a lack of LA capture.

superior and inferior PVs are initially created under guidance of double Lasso catheters and a 3D mapping system. Ablation is started at the superior wall and continued around the anterior and inferior venous perimeter. There is no vertical lesion line created at the posterior portion of the PVs along the esophageal aspect of the posterior LA. However, when the PVs are not isolated by only anterior lines, segmental ablation at the breakthrough points is performed (Figure 2). After complete isolation of all PVs, ablation of the LA roof is then performed by creating a contiguous line of ablation lesions joining the superior PVs. Finally, ablation of the LA floor is performed by creating a contiguous line of ablation lesions joining the inferior PVs to isolate the posterior LA.

Entrance block of the box lesion is confirmed by lack of potentials in the box during AF or sinus rhythm (Figure 3). Exit block of the box lesion is confirmed during sinus rhythm. Gaps along the ablation lines are detected and closed using high voltage $(10 \mathrm{~V})$ pace mapping through the ablation catheter (Figure 4). With lack of LA capture, the line is considered as complete at this location. In the case of LA capture, a gap is suspected and RF energy delivered simultaneously while pacing from the tip of the ablation catheter. The endpoint of box isolation is defined as bidirectional conduction block, that is, lack of potentials in the box and lack of LA capture. This is important to prevent a recurrence of gap-related atrial flutter.

\section{Ablation of CFAE}

PV isolation or box isolation alone is effective for treating paroxysmal $\mathrm{AF}$, but is not enough for curing persistent $\mathrm{AF}$ or longstanding persistent AF. ${ }^{31,32}$ To improve the clinical outcome, extensive ablation, including multiple linear lesions and/or ablation of CFAE, has been widely adopted, particu- larly in patients with persistent $\mathrm{AF}$ or longstanding persistent AF. ${ }^{7,33-36}$ Nademanee et $\mathrm{al}^{7}$ have provided a new electrogramguided approach by mapping and targeting areas of CFAE defined as fractionated electrograms composed of 2 or more deflections with a mean cycle length $\leq 120 \mathrm{~ms}$. CFAE may indicate slow conduction, pivot points of wave fronts, reentries and drivers. Also, there is a close relationship between autonomic ganglionated plexi activity and CFAE. ${ }^{8}$

Nademanee et $\mathrm{al}^{37}$ showed that AF was terminated in over $85 \%$ of patients, and reported that after CFAE ablation, $93 \%$ of the patients with paroxysmal AF, $87 \%$ of patients with persistent $\mathrm{AF}$ and $78 \%$ of patients with longstanding persistent AF were arrhythmia-free, including $11 \%$ of the patients taking antiarrhythmic drugs. However, their results were not fully reproduced by others. Oral et $\mathrm{al}^{34}$ performed ablation of CFAE in patients with chronic $\mathrm{AF}$ and in their study, only $12 \%$ of the patients had AF converted to sinus rhythm during the ablation and $4 \%$ converted to atrial flutter. Only $33 \%$ of the patients were in sinus rhythm without antiarrhythmic drugs after a single procedure and $57 \%$ of the patients were in sinus rhythm after a second procedure. Although it is unclear what are the factors underlying the differences in outcome between the 2 studies, several reasons may explain them, including additional right atrial ablation, the power and duration of RF energy, and the study endpoint.

CFAE are sometimes recorded over a diffuse area and numerous ablation applications are often necessary to eliminate all CFAE or to terminate AF. It is also difficult to distinguish culprit CFAE (eg, CFAE associated with perpetuating AF) from bystander CFAE. Although the most robust endpoint may be termination of $\mathrm{AF}$, this generally requires a very long procedure time. ${ }^{35}$ Furthermore, extensive ablation is associated with procedural complications, proarrhythmia, ${ }^{36}$ stroke risk, and 
compromise of atrial mechanical function. ${ }^{38}$ Nademanee et $\mathrm{al}^{37}$ used intravenous ibutilide to highlight the CFAE associated with perpetuating AF after CFAE ablation, if the patient remains in AF despite elimination of all visible CFAE. They demonstrated that ablation of CFAE resulted in termination of $\mathrm{AF}$ in $95 \%$ of patients; however, $28 \%$ required concomitant ibutilide treatment. ${ }^{37}$

\section{Long-Term Efficacy of AF Ablation}

Recently, 2 5-year follow-up studies reported that single-procedure success rates without antiarrhythmic drugs were $47 \%$ in patients with paroxysmal $\mathrm{AF}^{39}$ and $29 \%$ in patients with all types of $\mathrm{AF},{ }^{40}$ and after a repeat procedure, the success rates increased to $80 \%$ and $63 \%$, respectively.

At the Heart Rhythm Center, 513 patients, including 353 with paroxysmal $\mathrm{AF}, 73$ with persistent $\mathrm{AF}(<1$ year, mean $5 \pm 2$ months), and 87 with longstanding persistent $\mathrm{AF}(\geq 1$ year, mean $5 \pm 4$ years) underwent box isolation, after which, CFAE ablation was performed in $34(47 \%)$ patients with persistent $\mathrm{AF}$, and $70(80 \%)$ patients with longstanding persistent $\mathrm{AF}$. After a single ablation procedure, AF recurred in 70 (20\%) patients with paroxysmal AF, 20 (27\%) patients with persistent $\mathrm{AF}$, and $31(36 \%)$ patients with longstanding persistent $\mathrm{AF}$. In the patients with $\mathrm{AF}$ recurrence, antiarrhythmic drugs were re-administered. A second ablation procedure was performed in $39(11 \%)$ patients with paroxysmal AF, $13(18 \%)$ patients with persistent $\mathrm{AF}$, and $26(30 \%)$ patients with longstanding persistent $\mathrm{AF}$, including atrial tachycardia or flutter in $3 \%$ of the patients. During the second session, recovered conduction gaps along the lines were found in $88 \%$ of the patients and re-box isolation was performed. Additional ablation was performed in $74 \%$ of the patients, including CFAE in $48 \%$, superior vena cava isolation in $30 \%$, mitral isthmus in $26 \%$, focal atrial tachycardia in $24 \%$, cavotricuspid isthmus in $20 \%$, and gap-related flutter in $11 \%$.

After a follow-up period of $24 \pm 8$ months, 328 (93\%) patients with paroxysmal AF, $65(89 \%)$ patients with persistent $\mathrm{AF}$ and $74(85 \%)$ patients with longstanding persistent $\mathrm{AF}$ were free of AF, of whom 297 (84\%) patients with paroxysmal AF, $58(79 \%)$ patients with persistent AF, and $63(72 \%)$ patients with longstanding persistent AF were without antiarrhythmic drugs.

\section{Procedure Complications}

Catheter ablation of AF is associated with significant complications, ${ }^{41,42}$ which include vascular complications secondary to venous access, cardiac perforation/tamponade, valvular injury, embolic stroke or systemic embolism, PV stenosis, esophageal injury, and proarrhythmia resulting from reentrant tachycardias occurring from incomplete ablative lesions. The incidence of complications depends on operator experience.

At the Heart Rhythm Center, cardiac tamponade has occurred in 5 of 513 patients $(1.0 \%)$ and was managed by percutaneous drainage; 1 patient had homonymous hemianopsia; 1 patient had phrenic nerve injury, but recovered fully within 3 months; 1 patient had gastric hypomotility, but recovered fully within 2 weeks. No cases of atrio-esophageal fistula, significant PV stenosis or procedure-related death occurred.

\section{Indication of AF Ablation}

Ablation should be considered for patients with symptomatic paroxysmal $\mathrm{AF}$ that has failed treatment with one or more antiarrhythmic drugs, with normally sized or mildly dilated atria, and normal or mildly reduced ventricular function. Ablation may particularly benefit younger patients with lone AF who are frequently symptomatic and for whom very long-term antiarrhythmic and anticoagulation therapy has higher risks and costs.

It has been shown that good success of ablation can be achieved in patients with left ventricular dysfunction, ${ }^{43,44}$ previous cardiac surgery or valvular heart disease, ${ }^{45}$ and advanced age. ${ }^{46}$ Patients with heart failure benefit from AF ablation because the ejection fraction and functional endpoints such as exercise tolerance may significantly improve. ${ }^{44,47}$ Considering the potential of AF ablation to achieve rhythm control in symptomatic patients with paroxysmal AF and minimal or no organic heart disease, and the relative safety of the technique when performed by experienced operators, ablation may be considered as an initial therapy in selected patients.

There are several factors for determining the indication of AF ablation: the stage of atrial disease (ie, AF type, LA dimensions, duration of AF), the presence and severity of organic heart disease, age and the severity of symptoms, and patient preference. In symptomatic paroxysmal and persistent AF in patients with relevant organic heart disease, successful ablation is more difficult to achieve. In addition, little information is yet available about the late success of ablation in patients with heart failure and other advanced structural heart disease. ${ }^{48}$ Ablation of persistent and longstanding persistent AF is associated with variable but encouraging success rates, but often requires several attempts.

In clinical practice, many patients with AF may be asymptomatic but seek catheter ablation as an alternative to long-term anticoagulation. Although retrospective studies have demonstrated that discontinuation of oral anticoagulation therapy after ablation may be safe over the medium-term in some subsets of patients, this has never been confirmed by a large prospective randomized clinical trial and therefore remains unproven. ${ }^{49-51}$ Furthermore, it is well recognized that symptomatic and/or asymptomatic AF may recur during long-term follow-up after an AF ablation procedure. Therefore, discontinuation of anticoagulation therapy post ablation is generally not recommended in patients who have a CHADS score $\geq 2$. A patient's desire to eliminate the need for long-term anticoagulation by itself should not be considered an appropriate selection criterion.

\section{Meta-Analysis and Randomized Trials of Ablation vs. Antiarrhythmic Drugs}

Meta-analyses of studies performed mostly in patients with paroxysmal AF have confirmed the superiority of catheter ablation compared with antiarrhythmic medication. ${ }^{52-56}$ However, there is no evidence so far that successful AF ablation will result in reduced mortality, but a large prospective worldwide trial is already underway: catheter ablation vs. antiarrhythmic drug therapy for AF (CABANA). It is conceivable that AF ablation embedded in a comprehensive rhythm control intervention is most effective and beneficial when performed early during the course of disease..$^{57}$

\section{Conclusions}

AF is an arrhythmia associated with increased morbidity and mortality. Current therapies, especially antiarrhythmic drugs, not only are ineffective but can threaten a patient's quality of life and even longevity. AF ablation is an effective, safe, and established treatment that offers an excellent chance of a last- 
ing cure. Therefore, it is about time that $\mathrm{AF}$ ablation became a first-line therapy for selected patients with AF in terms of efficacy and safety when performed by experienced operators. $\mathrm{AF}$ ablation requires operator skills in manipulating catheters, understanding all facets of clinical electrophysiology, and treating procedure-related complications.

The present reality will gradually develop, and advances such as robotically controlled catheters and real-time MRI or CT imaging will help electrophysiologists to become more proficient in the task. AF ablation should be performed in centers that are well-equipped with an advanced mapping system and an experienced team to achieve the excellent outcomes.

\section{References}

1. Haissaguerre M, Jais P, Shah DC, Takahashi A, Hocini M, Quiniou $\mathrm{G}$, et al. Spontaneous initiation of atrial fibrillation by ectopic beats originating in the pulmonary veins. N Engl J Med 1998; 339: 659666.

2. Chen SA, Hsieh MH, Tai CT, Tsai CF, Prakash VS, Yu WC, et al. Initiation of atrial fibrillation by ectopic beats originating from the pulmonary veins: Electrophysiological characteristics, pharmacological responses, and effects of radiofrequency ablation. Circulation 1999; 100: 1879-1886.

3. Haissaguerre M, Shah DC, Jais P, Hocini M, Yamane T, Deisenhofer I, et al. Electrophysiological breakthroughs from the left atrium to the pulmonary veins. Circulation 2000; 102: 2463-2465.

4. Pappone C, Rosanio S, Oreto G, Tocchi M, Gugliotta F, Vicedomini $\mathrm{G}$, et al. Circumferential radiofrequency ablation of pulmonary vein ostia: A new anatomic approach for curing atrial fibrillation. Circulation 2000; 102: 2619-2628.

5. Hocini M, Jais P, Sanders P, Takahashi Y, Rotter M, Rostock T, et al. Techniques, evaluation, and consequences of linear block at the left atrial roof in paroxysmal atrial fibrillation: A prospective randomized study. Circulation 2005; 112: 3688-3696.

6. Kumagai K, Muraoka S, Mitsutake C, Takashima H, Nakashima H. A new approach for complete isolation of the posterior left atrium including pulmonary veins for atrial fibrillation. J Cardiovasc Electrophysiol 2007; 18: 1047-1052.

7. Nademanee K, McKenzie J, Kosar E, Schwab M, Sunsaneewitayakul B, Vasavakul T, et al. A new approach for catheter ablation of atrial fibrillation: Mapping of the electrophysiologic substrate. J Am Coll Cardiol 2004; 43: 2054-2056.

8. Nakagawa H, Yokoyama K, Scherlag BJ, Katari V, Aoyama H, Foresti S, et al. Ablation of autonomic ganglia. In: Steinberg JS, Calkins H, Jais P, editors. A practical approach to catheter ablation of atrial fibrillation. 1st edn. Lippincott, Williams and Wilkins Publishing, 2008; 218-230.

9. Ito M, Yanaga T, Saeki K, Arita M, Ishihara M, Mashiba H. Studies on sino-caval conduction of the rabbit with microelectrodes. Jpn J Physiol 1964; 14: 439-449.

10. Mandapati R, Skanes A, Chen J, Berenfeld O, Jalife J. Stable microreentrant sources as a mechanism of atrial fibrillation in the isolated sheep heart. Circulation 2000; 101: 194-199.

11. Skanes AC, Mandapati R, Berenfeld O, Davidenko JM, Jalife J. Spatiotemporal periodicity during atrial fibrillation in the isolated sheep heart. Circulation 1998; 98: 1236-1248.

12. Arora R, Verheule S, Scott L, Navarrete A, Katari V, Wilson E, et al. Arrhythmogenic substrate of the pulmonary veins assessed by highresolution optical mapping. Circulation 2003; 107: 1816-1821.

13. Kumagai K, Ogawa M, Noguchi H, Yasuda T, Nakashima H, Saku $\mathrm{K}$. Electrophysiologic properties of pulmonary veins assessed using a multielectrode basket catheter. J Am Coll Cardiol 2004; 43: 2281 2289.

14. Oral H, Knight BP, Ozaydin M, Chugh A, Lai SW, Scharf C, et al. Segmental ostial ablation to isolate the pulmonary veins during atrial fibrillation: Feasibility and mechanistic insights. Circulation 2002; 106: $1256-1262$.

15. Scharf C, Sneider M, Case I, Chugh A, Lai SW, Pelosi F Jr, et al. Anatomy of the pulmonary veins in patients with atrial fibrillation and effects of segmental ostial ablation analyzed by computed tomography. J Cardiovasc Electrophysiol 2003; 14: 150-155.

16. Dill T, Neumann T, Ekinci O, Breidenbach C, John A, Erdogan A, et al. Pulmonary vein diameter reduction after radiofrequency catheter ablation for paroxysmal atrial fibrillation evaluated by contrastenhanced three-dimensional magnetic resonance imaging. Circulation 2003; 107: 845-850.
17. Yamane T, Date T, Kanzaki Y, Inada K, Matsuo S, Shibayama K, et al. Segmental pulmonary vein antrum isolation using the "large-size" lasso catheter in patients with atrial fibrillation. Circ J 2007; 71: $753-760$.

18. Ouyang F, Bansch D, Ernst S, Schaumann A, Hachiya H, Chen M, et al. Complete isolation of left atrium surrounding the pulmonary veins: New insights from the double-Lasso technique in paroxysmal atrial fibrillation. Circulation 2004; 110: 2090-2096.

19. Verma A, Marrouche NF, Natale A. Pulmonary vein antrum isolation: Intracardiac echocardiography-guided technique. J Cardiovasc Electrophysiol 2004; 15: $1335-1340$.

20. Calkins H, Reynolds MR, Spector P, Sondhi M, Xu Y, Martin A, et al. Treatment of atrial fibrillation with antiarrhythmic drugs or radiofrequency ablation: Two systematic literature reviews and metaanalyses. Circ Arrhythm Electrophysiol 2009; 2: 349-361.

21. Verma A, Natale A, Padanilam BJ, Prystowsky EN. Why atrial fibrillation ablation should be considered first-line therapy for some patients. Circulation 2005; 112: 1214-1222.

22. Kamino K. Optical approaches to ontogeny of electrical activity and related functional organization during early heart development. Physiol Rev 1991; 71: 53-91.

23. Mandapati R, Skanes A, Chen J, Berenfeld O, Jalife J. Stable macroreentrant source as a mechanism of atrial fibrillation in the isolated sheep heart. Circulation 2000; 101: 194-199.

24. Lim WS, Tai CT, Hsieh MH, Tsai CF, Lin YK, Tsai HM, et al. Catheter ablation of paroxysmal atrial fibrillation initiated by nonpulmonary vein ectopy. Circulation 2003; 107: 3176-3183.

25. Marcids V, Schilling RJ, Ho SY, Chow AW, Davies DW, Peters NS. Characterization of left atrial activation in the intact human heart. Circulation 2003; 107: 733-739.

26. Todd DM, Skanes AC, Guiraudon G, Guiraudon C, Krahn AD, Yee $\mathrm{R}$, et al. Role of the posterior left atrium and pulmonary veins in human lone atrial fibrillation: Electrophysiological and pathological data from patients undergoing atrial fibrillation surgery. Circulation 2003; 108: $3108-3114$.

27. Sueda T, Nagata H, Rehash K, Morita S, Okada K, Sushi M, et al. Efficacy of a simple left atrial procedure for chronic atrial fibrillation in mitral valve operations. Ann Thorac Surg 1997; 63: 1070-1075.

28. Katritsis DG, Giazitzoglou E, Zografos T, Pokushalov E, Po SS, Camm AJ. Rapid pulmonary vein isolation combined with autonomic ganglia modification: A randomized study. Heart Rhythm 2011; 8: 672-678.

29. Pappone C, Santinelli V, Manguso F, Vicedomini G, Gugliotta F, Augello G, et al. Pulmonary vein denervation enhances long-term benefit after circumferential ablation for paroxysmal atrial fibrillation. Circulation 2004; 109: 327-334.

30. Yamaguchi Y, Kumagai K, Nakashima H, Saku K. Long-term effects of box isolation on sympathovagal balance in atrial fibrillation. Circ J 2010; 74: 1096-1103.

31. Kumagai K, Nakashima H. Noncontact mapping-guided catheter ablation of atrial fibrillation. Circ J 2009; 73: 233-241

32. Calkins H, Brugada J, Packer DL, Cappato R, Chen SA, Crijns HJ, et al. HRS/EHRA/ECAS expert consensus statement on catheter and surgical ablation of atrial fibrillation: Recommendations for personnel, policy, procedures and follow-up: A report of the Heart Rhythm Society (HRS) Task Force on catheter and surgical ablation of atrial fibrillation. Heart Rhythm 2007; 4: 816-861.

33. Haïssaguerre M, Sanders P, Hocini M, Hsu LF, Shah DC, Scavée C, et al. Changes in atrial fibrillation cycle length and inducibility during catheter ablation and their relation to outcome. Circulation 2004; 109: 3007-3013.

34. Oral H, Chugh A, Good E, Wimmer A, Dey S, Gadeela N, et al. Radiofrequency catheter ablation of chronic atrial fibrillation guided by complex electrograms. Circulation 2007; 115: 2606-2612.

35. Haïssaguerre M, Sanders P, Hocini M, Takahashi Y, Rotter M, Sacher $\mathrm{F}$, et al. Catheter ablation of long-lasting persistent atrial fibrillation: Critical structures for termination. J Cardiovasc Electrophysiol 2005; 16: $1125-1137$.

36. Haïssaguerre M, Hocini M, Sanders $\mathrm{P}$, Sacher F, Rotter M, Takahashi Y, et al. Catheter ablation of long-lasting persistent atrial fibrillation: Clinical outcome and mechanisms of subsequent arrhythmias. J Cardiovasc Electrophysiol 2005; 16: 1138-1147.

37. Nademanee K, Lockwood E, Oketani N, Gidney B. Catheter ablation of atrial fibrillation guided by complex fractionated atrial electrogram mapping of atrial fibrillation substrate. J Cardiol 2010; 55: 404408.

38. Lemola K, Desjardins B, Sneider M, Case I, Chugh A, Good E, et al. Effect of left atrial circumferential ablation for atrial fibrillation on left atrial transport function. Heart Rhythm 2005; 2: 923 -928.

39. Ouyang F, Tilz R, Chun J, Schmidt B, Wissner E, Zerm T, et al. 
Long-term results of catheter ablation in paroxysmal atrial fibrillation: Lessons from a 5-year follow-up. Circulation 2010; 122: 2368 2377.

40. Weerasooriya R, Khairy P, Litalien J, Macle L, Hocini M, Sacher F, et al. Catheter ablation for atrial fibrillation: Are results maintained at 5 years of follow-up? J Am Coll Cardiol 2011; 57: 160-166.

41. Cappato R, Calkins H, Chen SA, Davies W, Iesaka Y, Kalman J, et al. Worldwide survey on the methods, efficacy, and safety of catheter ablation for human atrial fibrillation. Circulation 2005; 111: $1100-1105$

42. Cappato R, Calkins H, Chen SA, Davies W, Iesaka Y, Kalman J, et al. Prevalence and causes of fatal outcome in catheter ablation of atrial fibrillation. J Am Coll Cardiol 2009; 53: 1798-1803.

43. Chen MS, Marrouche NF, Khaykin Y, Gillinov AM, Wazni O, Martin $\mathrm{DO}$, et al. Pulmonary vein isolation for the treatment of atrial fibrillation in patients with impaired systolic function. $\mathrm{J} \mathrm{Am} \mathrm{Coll} \mathrm{Cardiol}$ 2004; 43: 1004-1009.

44. Hsu LF, Jais P, Sanders P, Garrigue S, Hocini M, Sacher F, et al. Catheter ablation for atrial fibrillation in congestive heart failure. N Engl J Med 2004; 351: 2373-2383.

45. Khaykin Y, Marrouche NF, Saliba W, Schweikert R, Bash D, Chen MS, et al. Pulmonary vein antrum isolation for treatment of atrial fibrillation in patients with valvular heart disease or prior open heart surgery. Heart Rhythm 2004; 1: 33-39.

46. Bhargava M, Marrouche NF, Martin DO, Schweikert RA, Saliba W, Saad EB, et al. Impact of age on the outcome of pulmonary vein isolation for atrial fibrillation using circular mapping technique and cooled-tip ablation catheter. J Cardiovasc Electrophysiol 2004; 15: $8-13$.

47. Khan MN, Jais P, Cummings J, Di Biase L, Sanders P, Martin DO, et al. Pulmonary-vein isolation for atrial fibrillation in patients with heart failure. N Engl J Med 2008; 359: 1778-1785.

48. Callans DJ. Apples and oranges: Comparing antiarrhythmic drugs and catheter ablation for treatment of atrial fibrillation. Circulation 2008; 118: $2488-2490$
49. Oral H, Chugh A, Ozaydin M, Good E, Fortino J, Sankaran S, et al. Risk of thromboembolic events after percutaneous left atrial radiofrequency ablation of atrial fibrillation. Circulation 2006; 114: 759765.

50. Nademanee K, Schwab MC, Kosar EM, Karwecki M, Moran MD, Visessook N, et al. Clinical outcomes of catheter substrate ablation for high-risk patients with atrial fibrillation. J Am Coll Cardiol 2008; 51: $843-849$.

51. Themistoclakis S, Corrado A, Marchlinski FE, Jais P, Zado E, Rossillo A, et al. The risk of thromboembolism and need for oral anticoagulation after successful atrial fibrillation ablation. J Am Coll Cardiol 2010; 55: 735-743.

52. Wazni OM, Marrouche NF, Martin DO, Verma A, Bhargava M, SalibaW, et al. Radiofrequency ablation vs antiarrhythmic drugs as first-line treatment of symptomatic atrial fibrillation: A randomized trial. JAMA 2005; 293: 2634-2640.

53. Pappone C, Augello G, Sala S, Gugliotta F, Vicedomini G, Gulletta $\mathrm{S}$, et al. A randomized trial of circumferential pulmonary vein ablation versus antiarrhythmic drug therapy in paroxysmal atrial fibrillation: The APAF Study. J Am Coll Cardiol 2006; 48: 2340-2347.

54. Noheria A, Kumar A, Wylie JV Jr, Josephson ME. Catheter ablation vs antiarrhythmic drug therapy for atrial fibrillation: A systematic review. Arch Intern Med 2008; 168: 581-586.

55. Jais P, Cauchemez B, Macle L, Daoud E, Khairy P, Subbiah R, et al. Catheter ablation versus antiarrhythmic drugs for atrial fibrillation: The A4 study. Circulation 2008; 118: 2498-2505.

56. Wilber DJ, Pappone C, Neuzil P, De Paola A, Marchlinski F, Natale A, et al. Comparison of antiarrhythmic drug therapy and radiofrequency catheter ablation in patients with paroxysmal atrial fibrillation: A randomized controlled trial. JAMA 2010; 303: 333-340.

57. Kirchhof P, Bax J, Blomstrom-Lundquist C, Calkins H, Camm AJ, Cappato R, et al. Early and comprehensive management of atrial fibrillation: Executive summary of the proceedings from the $2^{\text {nd }}$ AFNET-EHRA consensus conference 'Research perspectives in AF'. Eur Heart J 2009; 30: 2969-2977. 